\title{
Relativistic and nonrelativistic annihilation of dark matter: a sanity check using an effective field theory approach
}

\author{
Mirco Cannoni ${ }^{\mathrm{a}}$ \\ Departamento de Física Aplicada, Facultad de Ciencias Experimentales, Universidad de Huelva, 21071 Huelva, Spain
}

Received: 29 June 2015 / Accepted: 1 March 2016 / Published online: 11 March 2016

(C) The Author(s) 2016. This article is published with open access at Springerlink.com

\begin{abstract}
We find an exact formula for the thermally averaged cross section times the relative velocity $\left\langle\sigma v_{\text {rel }}\right\rangle$ with relativistic Maxwell-Boltzmann statistics. The formula is valid in the effective field theory approach when the masses of the annihilation products can be neglected compared with the dark matter mass and cut-off scale. The expansion at $x=m / T \gg 1$ directly gives the nonrelativistic limit of $\left\langle\sigma v_{\text {rel }}\right\rangle$, which is usually used to compute the relic abundance for heavy particles that decouple when they are nonrelativistic. We compare this expansion with the one obtained by expanding the total cross section $\sigma(s)$ in powers of the nonrelativistic relative velocity $v_{r}$. We show the correct invariant procedure that gives the nonrelativistic average $\left\langle\sigma_{\mathrm{nr}} v_{r}\right\rangle_{\mathrm{nr}}$ coinciding with the large $x$ expansion of $\left\langle\sigma v_{\text {rel }}\right\rangle$ in the comoving frame. We explicitly formulate flux, cross section, thermal average, collision integral of the Boltzmann equation in an invariant way using the true relativistic relative $v_{\text {rel }}$, showing the uselessness of the Møller velocity and further elucidating the conceptual and numerical inconsistencies related with its use.
\end{abstract}

\section{Introduction}

While there is compelling evidence in astrophysics and cosmology that most of the mass of the Universe is composed of a new form of non baryonic dark matter (DM), there is a lack of evidence for the existence of new physics at LHC and other particle physics experiments. On the theory side, many specific models with new particles and interactions beyond the standard model have been proposed to account for DM.

Under these circumstances where no clear indications in favor of a particular model are at our disposal, the phenomenology of DM as been studied in a model independent way using an effective field theory approach; see for example [1-23].

\footnotetext{
a e-mail: mirco.cannoni@dfa.uhu.es
}

Measurements of the parameters of standard model of cosmology [24,25] furnish the present day mass density of DM, the relic abundance, $\Omega h^{2} \sim 0.11$ with an uncertainty at the level of $1 \%$. Any model that pretends to account for DM must reproduce this number, which, on the other hand, sets strong constraints on the free parameters of the model.

When the DM particles are weakly interacting massive particles that decouple from the primordial plasma at a temperature when they are nonrelativistic, the relativistic averaged annihilation rate $\left\langle\sigma v_{\text {rel }}\right\rangle$ can be well approximated by taking the nonrelativistic average of the first two terms of the expansion of $\sigma$ in powers of the nonrelativistic relative velocity. With $v_{\text {rel }}$ we indicate the relativistic relative velocity and with $v_{r}$ the nonrelativistic relative velocity, as defined in Appendix B. To describe collisions in a gas, and in particular in the primordial plasma, the reference frame that matters is the comoving frame (COF) where the observer sees the gas at rest as a whole and the colliding particles have general velocities $\boldsymbol{v}_{1,2}$ without any further specification of the kinematics.

It is thus desirable to formulate cross sections and rates in a relativistic invariant way, such that all the formulas and nonrelativistic expansions are valid automatically in the COF. Obviously, invariant formulas give the same results in the lab frame (LF), the frame where one massive particle is at rest, and in the center of mass frame (CMF) where the total momentum is zero. We will see that the key for the invariant formulation is $v_{\text {rel }}$.

On the contrary, in DM literature [26] instead of $v_{\text {rel }}$ it is used the so-called Møller velocity $\bar{v}$; see Appendix B. That this is incorrect was already discussed in Ref. [27] but papers using $\bar{v}$ continue to appear. The problem with $\bar{v}$, which is not the relative velocity, is its non-invariant and non-physical nature, for it can take values larger than $c$.

In this paper we first find an exact formula for $\left\langle\sigma v_{\text {rel }}\right\rangle$ as a function of $x=m / T$ calculated with the relativistic Maxwell-Boltzmann statistics. The formula is valid in the 
effective field theory framework such that the masses of the annihilation products can be neglected compared with the DM and the cut-off scale. For concreteness we work with fermion DM. We find the thermal functions corresponding to various interactions and in particular those corresponding to $s$ and $p$ wave scattering in the nonrelativistic limit which is given by the expansion at $x \gg 1$. This is done in Sect. 2, and Appendix A contains some mathematical results needed for the derivation of the exact formula and its asymptotic expansions.

Then, in Sect. 3, we present the correct invariant method for obtaining the same expansion by expanding the total annihilation cross section $\sigma(s)$ in powers of $v_{r}$.

We then discuss in Sect. 4 the problems with the use $\bar{v}$, while the numerical impact on the relic abundance of some incorrect methods employed in the literature is evaluated in Sect. 5.

Appendix B is preparatory for the whole paper: we recall how relativistic flux, cross section, rate, collision term of the Boltzmann equation and thermal averaged rate can be defined in the invariant way in terms of $v_{\text {rel }}$ showing the uselessness of the Møller velocity.

\section{Exact formula for the thermal average in the effective approach}

We consider a DM fermion field $\chi$ that couples to other fermion fields $\psi$ through an effective dimension- 6 operator of the type

$\mathcal{L}_{\Lambda}=\frac{\lambda_{a} \lambda_{b}}{\Lambda^{2}}\left(\bar{\chi} \Gamma_{a} \chi\right)\left(\bar{\psi} \Gamma_{b} \psi\right)$

The DM particles can be of Dirac or Majorana nature and have mass $m$, while $\psi$ are the standard model fermions or new ones. Here $\lambda_{a, b}$ are dimensionless coupling associated with the interactions described by combination of Dirac matrices $\Gamma_{a, b} . \Lambda$ is the energy scale below which the effective field theory is valid. In the exact theory $\Lambda$ corresponds to the mass of a heavy scalar or vector boson mediator that appears in the propagators. The $\psi$ masses can be neglected compared to $\Lambda$ and $m$. The exchange of a heavy mediator with mass $\Lambda$ may take place in the $s$-channel and/or in $t$-channel, as depicted in Fig. 1, depending on the specific model.

\subsection{Exact formula for $\left\langle\sigma v_{\text {rel }}\right\rangle$}

In all generality, for $2 \rightarrow 2$ processes, the matrix elements depend only on two independent Mandelstam variables, for example $s$ and $t$, and the squared matrix element is dimensionless. After integrating over the CMF angle, for example, the only remaining dependence is on $s$ and $m$. Any amplitude
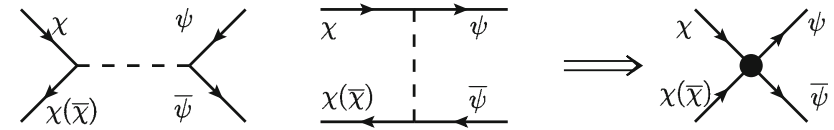

Fig. $1 s$ and $t$ channel annihilation diagrams reducing to the effective vertex corresponding to the lagrangian Eq. (1)

related to the operator (1) gives an integrated squared matrix element $\overline{|\mathcal{M}|^{2}}$ summed over the final spins and averaged over the initial spins that is a simple polynomial of the type

$w=\int \overline{|\mathcal{M}|^{2}} \mathrm{~d} \cos \theta=p_{2} s^{2}+p_{1} m^{2} s+p_{0} m^{4}$,

with $p_{0}, \ldots, p_{2}$ depending on $\Lambda$ and $\lambda_{a, b}$.

To get the formula for $\left\langle\sigma v_{\text {rel }}\right\rangle$ in a useful form, it is convenient to define the reduced cross section

$\sigma_{0}=\frac{1}{2 m^{2}} \frac{1}{32 \pi} w$

and the effective cross section

$\sigma_{\Lambda}=\frac{\lambda_{a}^{2} \lambda_{b}^{2}}{4 \pi} \frac{m^{2}}{\Lambda^{4}}$

which contains all the couplings. In terms of the effective cross section (4), and of the dimensionless variable $y=s /\left(4 m^{2}\right)$, the reduced cross section Eq. (3) becomes

$\sigma_{0}=\sigma_{\Lambda}\left(a_{2} y^{2}+\frac{a_{1}}{4} y+\frac{a_{0}}{16}\right)$,

where now $a_{2}, \ldots, a_{0}$ are pure numbers. The total unpolarized cross section then is

$\sigma=\frac{2 m^{2}}{s} \frac{\sqrt{\lambda\left(s, m_{3}^{2}, m_{4}^{2}\right)}}{\sqrt{\lambda\left(s, m_{1}^{2}, m_{2}^{2}\right)}} \sigma_{0}$

We now set $m_{1}=m_{2}=m, m_{3}=m_{4}=0$ in Eq. (6) and in Eq. (B.23), and we change variable to $y$. Thus Eq. (B.23) becomes

$\left\langle\sigma v_{\text {rel }}\right\rangle=\frac{2 x}{K_{2}^{2}(x)} \int_{1}^{\infty} \mathrm{d} y \sqrt{y-1} K_{1}(2 x \sqrt{y}) \sigma_{0}(y)$.

Using the integrals of Appendix A, we find

$$
\begin{aligned}
\left\langle\sigma v_{\text {rel }}\right\rangle=\sigma_{\Lambda} \frac{1}{16} & {\left[8 a_{2}+2 a_{1}+\left(5 a_{2}+2 a_{1}+a_{0}\right) \frac{K_{1}^{2}(x)}{K_{2}^{2}(x)}\right.} \\
& \left.+3 a_{2} \frac{K_{3}^{2}(x)}{K_{2}^{2}(x)}\right] .
\end{aligned}
$$


In the case $m_{3}=m_{4}=0$ the pure mass terms do not appear in the cross sections, thus $a_{0}=0$. Furthermore, we can relate $a_{2}$ and $a_{1}$ each other by an appropriate multiplicative factor,

$a_{1}=k a_{2}$,

and we express the cross sections as a function of $a_{2}$ only. The general formula (8) thus finally becomes

$\left\langle\sigma v_{\text {rel }}\right\rangle=\sigma_{\Lambda} a_{2} \mathcal{F}_{k}(x)$

with

$\mathcal{F}_{k}(x)=\frac{1}{16}\left(8+2 k+(5+2 k) \frac{K_{1}^{2}(x)}{K_{2}^{2}(x)}+3 \frac{K_{3}^{2}(x)}{K_{2}^{2}(x)}\right)$

the factored out thermal function.

The nonrelativistic thermal average is given by the expansion at $x \gg 1$. Using the asymptotic expansions Eq. (A.2) we find

$\left\langle\sigma_{\mathrm{nr}} v_{r}\right\rangle_{\mathrm{nr}}=\sigma_{\Lambda} a_{2}\left(1+\frac{k}{4}-\frac{3}{8} \frac{k}{x}\right)+\mathcal{O}\left(x^{-2}\right)$.

In the ultrarelativistic limit, $x \ll 1$, using the expansions (A.3), the thermal functions behave as $3 / x^{2}$, thus

$\left\langle\sigma v_{\mathrm{rel}}\right\rangle_{u r} \sim \sigma_{\Lambda} a_{2} \frac{3}{x^{2}}=\frac{\lambda_{a}^{2} \lambda_{b}^{2}}{4 \pi \Lambda^{4}} 3 a_{2} T^{2}$,

which is the expected result for massless particles.

The exact integration is possible because the effective operator removes the momentum dependence in the propagators that are reduced to a multiplicative constant and the assumption $m_{3}=m_{4}=0$ allows one to simplify the square $\operatorname{root} \sqrt{\lambda\left(s, m_{3}^{2}, m_{4}^{2}\right)}=s$ in the cross section (6). For example, with $m_{3}=m_{4}=m_{\psi}$, Eq. (7) becomes

$\left\langle\sigma v_{\text {rel }}\right\rangle=\frac{2 x}{K_{2}^{2}(x)} \int_{y_{0}}^{\infty} \mathrm{d} y \sqrt{y-\rho} \sqrt{y-1} K_{1}(2 x \sqrt{y}) \sigma_{0}(y, \rho)$,

with $\rho=m_{\psi}^{2} / m^{2}$ and $y_{0}=1$ if $m \geq m_{\psi}, y_{0}=\rho$ if $m<m_{\psi}$. In this case the exact integration is not possible but nonrelativistic expansions exist also in the case $\rho=1$ and $\rho \gg 1$ as we have shown in Ref. [27].

\subsection{Applications}

In order to show the thermal behavior of different interactions, we calculate the cross sections for various operators of the type (1), both for $s$ and $t$ channel annihilation. We list the quantity $\varpi=\Lambda^{4} /\left(\lambda_{a}^{2} \lambda_{b}^{2}\right) w$ and the resulting average Eq. (10).

For the s-channel annihilation we find:
1. Scalar: $(\bar{\chi} \chi)(\bar{\psi} \psi),(\bar{\chi} \chi)\left(\bar{\psi} \gamma^{5} \psi\right)$.

$\varpi=2 s\left(s-4 m^{2}\right), \quad\left\langle\sigma_{S} v_{\text {rel }}\right\rangle=\sigma_{\Lambda} 2 \mathcal{F}_{-4}(x)$.

2. Pseudoscalar: $\left(\bar{\chi} \gamma^{5} \chi\right)\left(\bar{\psi} \gamma^{5} \psi\right),\left(\bar{\chi} \gamma^{5} \chi\right)(\bar{\psi} \psi)$ :

$\varpi=2 s^{2}, \quad\left\langle\sigma_{\mathrm{PS}} v_{\text {rel }}\right\rangle=\sigma_{\Lambda} 2 \mathcal{F}_{0}(x)$.

3. Chiral: $\left(\bar{\chi} P_{L, R} \chi\right)\left(\bar{\psi} P_{L, R} \psi\right)$.

$\varpi=\frac{1}{2} s\left(s-2 m^{2}\right), \quad\left\langle\sigma_{C} v_{\text {rel }}\right\rangle=\sigma_{\Lambda} \frac{1}{2} \mathcal{F}_{-2}(x)$.

4. Pseudovector: $\left(\bar{\chi} \gamma^{\mu} \gamma_{5} \chi\right)\left(\bar{\psi} \gamma_{\mu} \gamma_{5} \psi\right),\left(\bar{\chi} \gamma^{\mu} \gamma_{5} \chi\right)\left(\bar{\psi} \gamma_{\mu} \psi\right)$.

$$
\varpi=\frac{8}{3} s\left(s-4 m^{2}\right), \quad\left\langle\sigma_{\mathrm{PV}} v_{\text {rel }}\right\rangle=\sigma_{\Lambda} \frac{8}{3} \mathcal{F}_{-4}(x)
$$

5. Vector: $\left(\bar{\chi} \gamma^{\mu} \chi\right)\left(\bar{\psi} \gamma_{\mu} \psi\right),\left(\bar{\chi} \gamma^{\mu} \chi\right)\left(\bar{\psi} \gamma_{\mu} \gamma^{5} \psi\right)$.

$$
\varpi=\frac{8}{3} s\left(s+2 m^{2}\right), \quad\left\langle\sigma_{V} v_{\text {rel }}\right\rangle=\sigma_{\Lambda} \frac{8}{3} \mathcal{F}_{2}(x) .
$$

6. Vector-chiral: $\left(\bar{\chi} \gamma^{\mu} P_{L, R} \chi\right)\left(\bar{\psi} \gamma_{\mu} P_{L, R} \psi\right)$.

$$
\varpi=\frac{8}{3} s\left(s-m^{2}\right), \quad\left\langle\sigma_{\mathrm{VC}} v_{\text {rel }}\right\rangle=\sigma_{\Lambda} \frac{8}{3} \mathcal{F}_{-1}(x) .
$$

The tensor interaction $\sigma^{\mu \nu}$ gives the same function as the vector case and is not reported. In the case of a Majorana $\chi$ clearly the vector and tensor interactions are absent, and the inclusion of a factor $1 / 2$ in the operator (1) cancels the factor 4 due to the presence of the exchange diagram of the initial identical particles.

Now we consider some examples of t-channel annihilation for operators common to Dirac and Majorana DM annihilation:

1. Scalar, pseudoscalar: $(\bar{\chi} \chi)(\bar{\psi} \psi),(\bar{\chi} \chi)\left(\bar{\psi} \gamma^{5} \psi\right)$,

$$
\left(\bar{\chi} \gamma^{5} \chi\right)\left(\bar{\psi} \gamma^{5} \psi\right),\left(\bar{\chi} \gamma^{5} \chi\right)(\bar{\psi} \psi) \text {. }
$$

$$
\begin{aligned}
& \varpi_{D}=\frac{2}{3} s\left(s-m^{2}\right), \quad\left\langle\sigma_{S, \mathrm{PS}}^{D, t} v_{\text {rel }}\right\rangle=\sigma_{\Lambda} \frac{2}{3} \mathcal{F}_{-1}(x) . \quad \text { (20) } \\
& \varpi_{M}=\frac{1}{3} s\left(5 s-14 m^{2}\right), \quad\left\langle\sigma_{S, \mathrm{PS}}^{M, t} v_{\text {rel }}\right\rangle=\sigma_{\Lambda} \frac{1}{3} \mathcal{F}_{-\frac{14}{5}}(x) .
\end{aligned}
$$

2. Chiral: $\left(\bar{\chi} P_{L, R} \chi\right)\left(\bar{\psi} P_{L, R} \psi\right)$.

$$
\begin{aligned}
& \varpi_{D}=\frac{1}{6} s\left(s-m^{2}\right), \quad\left\langle\sigma_{C}^{D, t} v_{\text {rel }}\right\rangle=\sigma_{\Lambda} \frac{1}{6} \mathcal{F}_{-1}(x) . \\
& \varpi_{M}=\frac{1}{3} s\left(s-4 m^{2}\right), \quad\left\langle\sigma_{C}^{M, t} v_{\text {rel }}\right\rangle=\sigma_{\Lambda} \frac{1}{3} \mathcal{F}_{-4}(x) .
\end{aligned}
$$




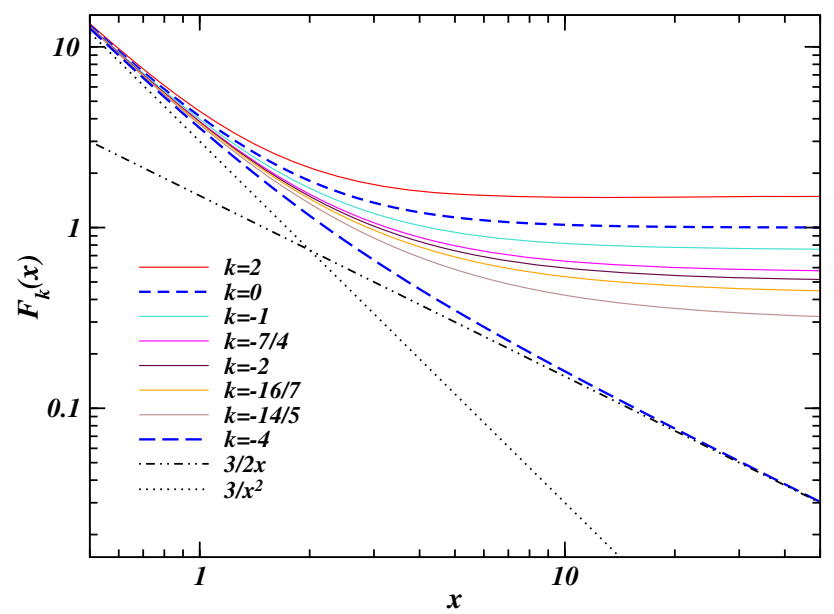

Fig. 2 The thermal function (11) for the interactions and annihilation cross sections considered in the text

3. Pseudovector: $\left(\bar{\chi} \gamma^{\mu} \gamma_{5} \chi\right)\left(\bar{\psi} \gamma_{\mu} \gamma_{5} \psi\right),\left(\bar{\chi} \gamma^{\mu} \gamma_{5} \chi\right)\left(\bar{\psi} \gamma_{\mu} \psi\right)$.

$$
\begin{aligned}
& \varpi_{D}=\frac{4}{3} s\left(4 s-7 m^{2}\right), \quad\left\langle\sigma_{\mathrm{PV}}^{D, t} v_{\text {rel }}\right\rangle=\sigma_{\Lambda} \frac{4}{3} \mathcal{F}_{-\frac{7}{4}}(x) . \\
& \varpi_{M}=\frac{8}{3} s\left(7 s-16 m^{2}\right), \quad\left\langle\sigma_{\mathrm{PV}}^{M, t} v_{\text {rel }}\right\rangle=\sigma_{\Lambda} \frac{8}{3} \mathcal{F}_{-\frac{16}{7}}(x) .
\end{aligned}
$$

The thermal functions corresponding to the previous cases are shown in Fig. 2 where the asymptotic behaviors are clearly seen. In particular we note that

$$
\begin{aligned}
\mathcal{F}_{0}(x) & =\frac{1}{16}\left(8+5 \frac{K_{1}^{2}(x)}{K_{2}^{2}(x)}+3 \frac{K_{3}^{2}(x)}{K_{2}^{2}(x)}\right), \\
\mathcal{F}_{-4}(x) & =\frac{3}{16}\left(-\frac{K_{1}^{2}(x)}{K_{2}^{2}(x)}+\frac{K_{3}^{2}(x)}{K_{2}^{2}(x)}\right),
\end{aligned}
$$

behave in the nonrelativistic limit as

$\mathcal{F}_{0}(x) \sim 1+\mathcal{O}\left(x^{-2}\right), \quad \mathcal{F}_{-4}(x) \sim \frac{3}{2 x}+\mathcal{O}\left(x^{-2}\right)$.

The function $\mathcal{F}_{0}(x)$, which appears in the $s$-channel annihilation through a pseudoscalar interaction, is the only case where the term of order $\mathcal{O}\left(x^{-1}\right)$ is absent, while $\mathcal{F}_{-4}(x)$, which appears in the scalar and axial-vector $s$-channel annihilation and in the chiral $t$-channel Majorana fermion annihilation, is the only case where the constant $\mathcal{O}\left(x^{0}\right)$ term is zero. These are the exact temperature dependent factors that correspond to the phenomenological interpolating functions proposed in Ref. [28] to model the $s$-wave and $p$-wave behavior in the nonrelativistic limit. For all other interactions both $s$-wave and $p$-wave contributions are present. The function $\mathcal{F}_{-4}(x)$ can also be read off from the formulas of Ref. [29] where the $t$-channel annihilation of Majorana fermions with the exchange of a scalar with chiral couplings was considered.

We note that although we have concentrated on the case of fermion DM, the formula is valid for DM scalar and vector candidates as well, with the necessary redefinition of $\sigma_{\Lambda}$.

\section{Expansion of the cross section in powers of the relative velocity}

In the general case $m_{3}=m_{4}=m_{\psi} \neq 0$ the exact integration is not possible. If the relative velocity of the annihilating particles is small compared with the velocity of light we can work directly with nonrelativistic formulas. The exothermic annihilation cross section in the nonrelativistic limit, to the lowest orders in $v_{r}$, is usually expanded as $\sigma_{\mathrm{nr}} \sim a / v_{r}+b v_{r}$, and multiplying by $v_{r}$,

$\sigma_{\mathrm{nr}} v_{r} \sim a+b v_{r}^{2}$.

Then, using Eqs. (B.21) and (B.22), the nonrelativistic thermal average of Eq. (28) is

$\left\langle\sigma_{\mathrm{nr}} v_{r}\right\rangle_{\mathrm{nr}} \sim a+6 \frac{b}{x}$.

In the case of our cross sections, comparing Eq. (29) with (12), the coefficients are thus ${ }^{1}$

$a=\sigma_{\Lambda} a_{2}\left(1+\frac{k}{4}\right), \quad b=-\sigma_{\Lambda} a_{2} \frac{k}{16}$.

We now ask, given $\sigma(s)$, how to perform the expansion in terms of the relative velocity to find the coefficients $a$ and $b$ that correspond to the large $x$ expansion of the relativistic thermal average in the COF. Combining Eqs. (5), (6), and (9), the general total annihilation cross section reads

$\sigma=\sigma_{\Lambda} \frac{a_{2}}{2} \frac{\sqrt{s}}{\sqrt{s-4 m^{2}}}\left(\frac{s}{4 m^{2}}+\frac{k}{4}\right)$.

1 This result must coincide with the expansion of Refs. [27,30]. With our notation the expansion is

$\left.\left\langle\sigma_{\mathrm{nr}} v_{r}\right\rangle_{\mathrm{nr}} \sim \sigma_{0}\right|_{y=1}+\frac{3}{x}\left(-\left.\sigma_{0}\right|_{y=1}+\left.\frac{1}{2} \sigma_{0}^{\prime}\right|_{y=1}\right)$,

where the prime indicate derivative respect to the variable $y$. Comparison with the expansion (29) requires one to identify

$\left.a \equiv \sigma_{0}\right|_{y=1}, \quad b \equiv \frac{1}{2}\left(\left.\frac{1}{2} \sigma_{0}^{\prime}\right|_{y=1}-\left.\sigma_{0}\right|_{y=1}\right)$.

Using Eq. (5) with $a_{0}=0$ and $a_{2}=k a_{1}$, it is easy to verify that one obtains again Eq. (12). 
The correct way to proceed is to use the invariant relation Eq. (B.8) with $m_{1}=m_{2}=m$ and to solve it for $s$ as a function of $v_{\text {rel }}$ :

$s=2 m^{2}\left(1+\frac{1}{\sqrt{1-v_{\mathrm{rel}}^{2}}}\right)$.

This formula is valid in every frame and substituted in Eq. (31) gives the exact dependence of the cross section on the relativistic relative velocity, $\sigma\left(v_{\text {rel }}\right)$. Then, if $v_{\text {rel }} \sim v_{r} \ll 1$, we can expand the obtained expression to the desired order in $v_{r}$ and the nonrelativistic average taken using Eq. (B.22) will coincide with the expansion of Eq. (10) for $x \gg 1$, that is, the expansion (12).

Equivalently, in order to find the expansion (28), we note that the squared roots in the annihilation cross section (31) imply that a term of order $v_{r}^{4}$ in $s$ will contribute to the order $v_{r}^{2}$ in $\sigma$. Thus we need to expand $s$, formula (32), at least to order $v_{r}^{4}$,

$s \sim 4 m^{2}+m^{2} v_{r}^{2}+\frac{3}{4} m^{2} v_{r}^{4}$.

Substituting Eq. (33) in (31) and performing the expansion in powers of $v_{r}$ it easy to find

$\sigma_{\mathrm{nr}} v_{r} \sim \sigma_{\Lambda} a_{2}\left(1+\frac{k}{4}-\frac{k}{16} v_{r}^{2}\right)$,

in agreement with (30).

In the case of coannihilations [31], for example when a DM particles scatter off another particle with different mass, the Mandelstam invariant takes the form

$s=\left(m_{1}-m_{2}\right)^{2}+2 m_{1} m_{2}\left(1+\frac{1}{\sqrt{1-v_{\mathrm{rel}}^{2}}}\right)$,

with the expansion

$s \sim\left(m_{1}-m_{2}\right)^{2}+m_{1} m_{2} v_{r}^{2}+\frac{3}{4} m_{1} m_{2} v_{r}^{4}$.

This procedure gives the correct expansion in the $\mathrm{COF}$ where the velocities $\boldsymbol{v}_{1,2}$ of the colliding particles are specified in this frame. Clearly, the same expansion with the same coefficients is obtained in the LF and in the CMF.

\section{The problems with the Møller velocity}

The simple outlined procedure has not been recognized in DM literature where, incorrectly, the Møller velocity $\bar{v}$, Eq. (B.25), instead of $v_{\text {rel }}$ is considered. As recalled in
Appendix B, $\bar{v}$ is a non-invariant, non-physical velocity. The expression of $\bar{v}$ in terms of $s$ is thus different in different frames and the expansion of $\sigma$ takes different values in different frames.

Before discussing the problems with the Møller velocity we note that if we take the limit $m_{f} \rightarrow 0$ in the analogous expansions published many papers [4-11], we do not reproduce the expansion (34). The reason is that in these papers the expansion of $s$ is truncated to the lowest order in $v_{r}^{2}$,

$s \sim 4 m^{2}+m^{2} v_{r}^{2}$.

If we substitute this in Eq. (31) and expand, we find

$\sigma_{\mathrm{nr}} v_{r} \sim \sigma_{\Lambda} a_{2}\left(1+\frac{k}{4}+\frac{12+k}{32} v_{r}^{2}\right)$,

with an incorrect coefficient $b$. Clearly the same wrong result is obtained truncating (33) to order $v_{r}^{2}$, whatever the frame in which $v_{r}$ is specified, CMF, LF or COF.

We now go back to the Møller velocity (B.25). Evaluated in the CMF taking $m_{1}=m_{2}=m$ reads

$\bar{v}_{*}=\frac{2}{\sqrt{s_{*}}} \sqrt{s_{*}-4 m^{2}}$.

We indicate the quantities evaluated in the CMF with a "*”. By inverting Eq. (39) we find

$s_{*}=\frac{4 m^{2}}{1-\frac{\bar{v}_{*}^{2}}{4}}$.

This relation is different from (32) and is often incorrectly identified as the relation between $s$ and the relative velocity in the CMF; see for example [10,31]. In fact, the expansion to order $\mathcal{O}\left(v_{r, *}^{4}\right)$ reads

$s_{*} \sim 4 m^{2}+m^{2} v_{r, *}^{2}+\frac{m^{2}}{4} v_{r, *}^{4}$.

When used in (31), it gives the following nonreltivistic expansion of the cross section:

$\sigma_{\mathrm{nr}} v_{r, *} \sim \sigma_{\Lambda} a_{2}\left(1+\frac{k}{4}+\frac{1}{4} v_{r, *}^{2}\right)$,

which is different from the correct expansion (34).

Other authors, see Refs, [26] and [20-23], perform the expansion with the Møller velocity evaluated in the rest frame of one particle. Indicating with " $\ell$ " the quantities in this frame, Eq. (B.25) becomes

$\bar{v}_{\ell}=\frac{\sqrt{s_{\ell}} \sqrt{s_{\ell}-4 m^{2}}}{s_{\ell}-2 m^{2}}$, 
and by inverting Eq. (43) we obtain

$s_{\ell}=2 m^{2}\left(1+\frac{1}{\sqrt{1-\bar{v}_{\ell}^{2}}}\right)$.

This expression is formally identical to Eq. (32), thus when $\bar{v}_{\ell} \sim v_{r, \ell}$ and $s_{\ell}$ is expanded up to the order $v_{r, \ell}^{4}$ we obtain the expansion $\sigma_{\mathrm{nr}} v_{r, \ell}$, which formally coincides with Eq. (34), with $v_{r, \ell}$ in place of $v_{r}$.

It should be clear that this is just a mathematical coincidence due to the fact that $\bar{v}$ reduces to $v_{\text {rel }}$ only when one of the two velocities $\boldsymbol{v}_{1,2}$ is zero as it is evident from the definitions Eqs. (B.5) and (B.25). In other words, the expansion found in Refs. [20-23] are correct because the authors have implicitly used the relative velocity, Eqs. (B.8) and (33).

We thus emphasize some common statements found in the DM literature and why they do not subsist:

1. In the relativistic Boltzmann equation the $v$ in $\sigma v$ is $\bar{v}$ and $\langle\sigma v\rangle$ must be calculated in the LF frame.

This is not true, as shown in detail in Ref. [27] and in Appendix B. Using $v_{\text {rel }}$ and recognizing the non-physical nature of $\bar{v}$, one works always with invariant quantities and the consistency of the relativistic and nonrelativistic formulas and expansions is obtained in the comoving frame without any further specification of the kinematics. The LF, also called the Møller frame in Ref. [23], cannot be a privileged frame for the relic abundances calculation also because for massless particles the rest frame does not exist.

2. The Møller velocity coincides with relative velocity in a frame where the velocities are collinear.

This is not true because, for example, in the CMF where the particles have velocities $v_{*}$, the Møller velocity is $2 v_{*}$ while the relative velocity is $2 v_{*} /\left(1+v_{*}^{2}\right)$. Note that the true relative velocity is never superluminal.

\section{Impact on the relic abundance}

Only in the case $k=-4$ the incorrect expansions (38) and (42) coincide, incidentally, with the expansion (34). While the lowest order coefficient $a$ turns out to be always the same, the coefficient $b$ is different in any other case. To illustrate the impact of $b$ on the value of the relic abundance we consider the case of the $s$-channel annihilation with vector interaction, Eq. (18), and the $s$-channel annihilation with a pseudoscalar exchange, Eq. (15). In the first case $k=2, a_{2}=8 / 3$, and the correct coefficients $a$ and $b$ are
$a_{V}=4 \sigma_{\Lambda}, \quad b_{V}=-\frac{\sigma_{\Lambda}}{3}$,

while the incorrect coefficient $b$ in (38) and (42) is

$b_{V_{1}}=\frac{7}{6} \sigma_{\Lambda}, \quad b_{V_{2}}=\frac{2}{3} \sigma_{\Lambda}$.

In the second case, $k=0$ and $a_{2}=2$, thus

$a_{P S}=2 \sigma_{\Lambda}, \quad b_{P S}=0$,

and the wrong $b$ coefficients are

$b_{P S_{1}}=\frac{3}{4} \sigma_{\Lambda}, \quad b_{P S_{2}}=\frac{1}{2} \sigma_{\Lambda}$.

We calculate the relic abundance following the exact theory of freeze out presented in Ref. [32]. We briefly recall the main points. Let $Y_{0}=45 /\left(4 \pi^{4}\right)\left(g_{\chi} / g_{s}\right) x^{2} K_{2}(x)$ be the initial equilibrium abundance (number density over the entropy density), with $g_{\chi}=2$ for spin $1 / 2$ fermions and $g_{s}$ the relativistic degrees of freedom associated with the entropy density. The function $Y_{1}(x)$ that gives the abundance up to the point $x_{*}$ where $Y_{1}(x)-Y_{0}(x)$ is maximal is

$Y_{1}(x)=(1+\delta(x)) Y_{0}(x)$,

$\delta(x)=\sqrt{1-\frac{x^{2}}{C\left\langle\sigma v_{\text {rel }}\right\rangle Y_{0}} \frac{1}{Y_{0}} \frac{\mathrm{d} Y_{0}}{\mathrm{~d} x}}-1$,

with $x_{*}$ given by the condition

$-\frac{1}{Y_{0}(x)} \frac{\mathrm{d} Y_{0}(x)}{\mathrm{d} x}=\frac{1}{\delta(x)} \frac{\mathrm{d} \delta(x)}{\mathrm{d} x}$ at $x=x_{*}$.

The abundance at $x>x_{*}$ is found by integrating numerically the usual equation

$\frac{\mathrm{d} Y}{\mathrm{~d} x}=\frac{C}{x^{2}}\left\langle\sigma v_{\mathrm{rel}}\right\rangle\left(Y_{0}^{2}-Y^{2}\right)$,

with the initial condition $\left(x_{*}, Y\left(x_{*}\right)=Y_{1}\left(x_{*}\right)\right)$. The factor $C$ is defined by $C=\sqrt{\frac{\pi}{45}} M_{P} m_{\chi} \sqrt{g_{*}}$, where $M_{P}$ is the Planck mass and $\sqrt{g_{*}}=g_{s} / \sqrt{g_{\rho}}\left(1+T / 3 \mathrm{~d}\left(\ln g_{s}\right) / \mathrm{d} T\right)$ accounts for the temperature dependence of the relativistic degrees of freedom associated with the energy density, $g_{\rho}$, and $g_{s}[26$, 30]. For WIMP masses larger than $10 \mathrm{GeV}$ we can neglect the temperature dependence of the degrees of freedom $[33,34]$ and take $g_{s}=g_{\rho}=g=100, \sqrt{g_{*}}=\sqrt{g}$. In solving numerically (51) and (52) with the exposed method, we use the exact formula for $\left\langle\sigma v_{\text {rel }}\right\rangle$, Eq. (10).

We compare the previous numerical solution with the one obtained using the nonrelativistic freeze out approximation (FOA), which is commonly employed in the literature. The 
FOA consists in integrating Eq. (52) with an initial condition $\left(x_{f}, Y\left(x_{f}\right)\right)$ such that the equilibrium term proportional $Y_{0}^{2}$ can be neglected. We choose the freeze out point at the point $x_{2}$ where $Y\left(x_{2}\right) \simeq Y_{1}\left(x_{2}\right)=2 Y_{0}\left(x_{2}\right)$. As shown in Ref. [32], $Y_{1}(x)$ well approximates the true abundance also in the interval $x_{*}<x<x_{2} . x_{2}$ is the optimal point for the FOA and corresponds to the temperature where the extent of the inverse creation reaction $\psi \bar{\psi} \rightarrow \chi \chi$ is maximal. The solution in the freeze out approximation is then

$Y_{\mathrm{FOA}}=\frac{2 Y_{0}\left(x_{2}\right)}{1+2 Y_{0}\left(x_{2}\right) \frac{C}{x_{2}}\left(a+3 \frac{b}{x_{2}}\right)}$.

The freeze out point $x_{2}$ is given by the condition $-\frac{1}{Y_{0}} \frac{\mathrm{d} Y_{0}}{\mathrm{~d} x}=$ $3 \frac{C}{x^{2}}\left\langle\sigma v_{\text {rel }}\right\rangle Y_{0}$, which, in terms of the method of Ref. [35] corresponds to $c(c+2)=3$, that is, $c=1$. Using the nonrelativistic form of $Y_{0}$,

$Y_{0}=\frac{45}{4 \pi^{4}} \frac{g_{\chi}}{g_{s}} \sqrt{\frac{\pi}{2}} x^{3 / 2} e^{-x}$,

$x_{2}$ is given by the root of

$3 C\left(a+6 \frac{b}{x}\right) \sqrt{\frac{\pi}{2}} x^{-1 / 2} e^{-x}=1$.

Calling $\alpha=3 a C \sqrt{\pi / 2}$, an accurate analytical approximate solution of Eq. (55) is given by

$x_{2}=\ln \alpha-\frac{1}{2} \ln (\ln \alpha)+\ln \left(1+\frac{6 b}{a}(\ln \alpha)^{-1}\right)$.

The relic abundance normalized over the critical density is $\Omega h^{2}=2.755 \times 10^{8}(\mathrm{~m} / \mathrm{GeV}) Y_{(\infty)}$ for a Majorana fermion and two times that quantity for a Dirac fermion with the same density of antiparticles. We now compare the exact relic abundance $\Omega h^{2}$ with the value $\left(\Omega h^{2}\right)_{\text {FOA }}$ furnished by the nonrelativistic FOA calculated using the correct and the wrong expansions. We take the couplings $\lambda_{a, b}=1$ for illustrative purposes and two values of the cut off scale, $\Lambda=1,10$ $\mathrm{TeV}$. The value of the freeze out points $x_{*}$ and $x_{2}$ varies roughly between 18 and 30 in the parameter space with $m<\Lambda$ where the effective treatment is supposed to be valid. The ratio $\Omega h^{2} /\left(\Omega h^{2}\right)_{\mathrm{FOA}}$ is shown in Fig. 3 as a function of the DM mass for the chosen examples. The bottom blue curves show that the FOA with the correct coefficients (45) and (47) underestimates the numerical value by less than $2 \%$, and that in most part of the parameter space the error is at the level of $1 \%$ or less. This a test of goodness for our FOA, and confirms what shown in Ref. [32]. The red and the black curves show the effect of the wrong coefficients (46) and (48), respectively. The wrong expansions underestimate the relic

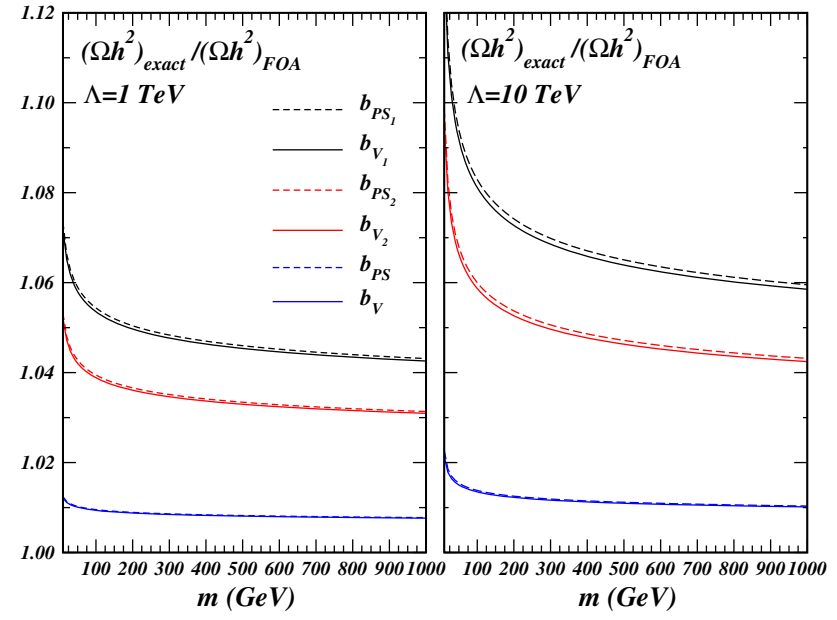

Fig. 3 Ratio of relic abundance obtained by solving numerically the abundance equation (52) over the value given by the freeze out approximation for the pseudoscalar and vector interactions. In the bottom blue curves for the FOA the correct coefficients (45) and (47) are used. The red and black lines show the effect of the wrong coefficients (46) and (48) respectively

abundance by a factor between 3 and $12 \%$ for both interactions for masses larger than $10 \mathrm{GeV}$ as shown in the plot. The behavior is similar for the other interactions not shown in figure. The error becomes even larger at smaller masses and we have verified that using for example $c=1 / 2$ and other values we get even worst approximations. Clearly this kind of error nowadays is not compatible with the precision with which the experimental value is known.

Acknowledgments This work was supported by a Grant under the MINECO/FEDER project: SOM: Sabor y origen de la Materia (CPI14-397). The author acknowledges Roberto Ruiz de Austri, Nuria Rius and Pilar Hernandez for hospitality and for useful discussions at the Instituto de Fisica Corpuscolar (IFIC) in Valencia where part of this work was done.

Open Access This article is distributed under the terms of the Creative Commons Attribution 4.0 International License (http://creativecomm ons.org/licenses/by/4.0/), which permits unrestricted use, distribution, and reproduction in any medium, provided you give appropriate credit to the original author(s) and the source, provide a link to the Creative Commons license, and indicate if changes were made.

Funded by SCOAP ${ }^{3}$.

\section{Appendix A: Integrals and expansions}

Equation (7) can be written as

$\left\langle\sigma v_{\text {rel }}\right\rangle=\sigma_{\Lambda} \frac{2 x}{K_{2}^{2}(x)}\left(a_{2} \mathrm{~A}_{2}+\frac{a_{1}}{4} \mathrm{~A}_{1}+\frac{a_{0}}{16} \mathrm{~A}_{0}\right)$.

The integrals are evaluated with methods similar to those described in Ref. [27] in terms of Bessel functions of the second kind: 


$$
\begin{aligned}
\mathrm{A}_{0} & =\int_{1}^{\infty} \mathrm{d} y \sqrt{y-1} K_{1}(2 x \sqrt{y})=\frac{1}{2 x} K_{1}^{2}(x) \\
\mathrm{A}_{1} & =\int_{1}^{\infty} \mathrm{d} y \sqrt{y-1} y K_{1}(2 x \sqrt{y})=\frac{1}{2 x} \frac{K_{1}^{2}(x)+K_{2}^{2}(x)}{2}, \\
\mathrm{~A}_{2} & =\int_{1}^{\infty} \mathrm{d} y \sqrt{y-1} y^{2} K_{1}(2 x \sqrt{y}) \\
& =\frac{1}{2 x} \frac{1}{16}\left[5 K_{1}^{2}(x)+8 K_{2}^{2}(x)+3 K_{3}^{2}(x)\right] .
\end{aligned}
$$

The expansions at $x \gg 1$ are

$$
\frac{K_{1}^{2}(x)}{K_{2}^{2}(x)} \sim 1-\frac{3}{x}+\mathcal{O}\left(x^{-2}\right), \quad \frac{K_{3}^{2}(x)}{K_{2}^{2}(x)} \sim 1+\frac{5}{x}+\mathcal{O}\left(x^{-2}\right),
$$

while for $x \ll 1$ are

$$
\frac{K_{1}^{2}(x)}{K_{2}^{2}(x)} \sim \frac{x^{2}}{4}+\mathcal{O}\left(x^{3}\right), \quad \frac{K_{3}^{2}(x)}{K_{2}^{2}(x)} \sim \frac{16}{x^{2}}+\mathcal{O}\left(x^{2}\right)
$$

\section{Appendix B: Invariant formulation using $v_{\text {rel }}$}

In this appendix we recall, based on the results of Ref. [27], the main points about the relation between the relative velocity, the Møller velocity, flux and thermal average which are used in the main text.

Appendix B.1: Invariant relative velocity

The relativistic relative velocity that generalizes the nonrelativistic relative velocity

$v_{r}=\left|\boldsymbol{v}_{1}-\boldsymbol{v}_{2}\right|$

is given by

$v_{\mathrm{rel}}=\frac{\sqrt{\left(\boldsymbol{v}_{1}-\boldsymbol{v}_{2}\right)^{2}-\frac{\left(\boldsymbol{v}_{1} \times \boldsymbol{v}_{2}\right)^{2}}{c^{2}}}}{1-\frac{\boldsymbol{v}_{1} \cdot \boldsymbol{v}_{2}}{c^{2}}}$.

We have explicitly written the dependence on the velocity of light $c$ to make manifest that $v_{\text {rel }}$ coincide with $v_{r}$ in the nonrelativistic limit because the scalar and vector products are of order $(v / c)^{2}$. In the following we go back to natural units.

The relative velocity $v_{\text {rel }}$ can be written using the Mandelstam invariant $s=\left(p_{1}+p_{2}\right)^{2}$, where $p_{1,2}$ are the fourmomenta, and $\lambda$, the Mandelstam triangular function,

$\lambda\left(s, m_{1}^{2}, m_{2}^{2}\right)=\left[s-\left(m_{1}+m_{2}\right)^{2}\right]\left[s-\left(m_{1}-m_{2}\right)^{2}\right]$, in a generic frame,

$$
\begin{aligned}
v_{\text {rel }} & =\frac{\sqrt{\left(p_{1} \cdot p_{2}\right)^{2}-m_{1}^{2} m_{2}^{2}}}{p_{1} \cdot p_{2}} \\
& =\frac{\sqrt{\lambda\left(s, m_{1}^{2}, m_{2}^{2}\right)}}{s-\left(m_{1}^{2}+m_{2}^{2}\right)},
\end{aligned}
$$

showing its invariant nature.

\section{Appendix B.2: Flux factor}

Given two bunches of particles with number densities $n_{1,2}$ and velocities $\boldsymbol{v}_{1,2}$ in a generic inertial frame, in nonrelativistic physics the flux is $F_{\mathrm{nr}}=n_{1} n_{2} v_{r}$. To obtain the relativistic invariant flux that reduces to $F_{\mathrm{nr}}$ in the nonrelativistic limit, the easiest way is to consider the 4-currents $J_{i}=\left(n_{i}, n_{i} \boldsymbol{v}_{i}\right)$, thus

$F=\left(J_{1} \cdot J_{2}\right) v_{\text {rel }}=n_{1} n_{2}\left(1-\boldsymbol{v}_{1} \cdot \boldsymbol{v}_{2}\right) v_{\text {rel }}$.

Note that the factor $\left(1-\boldsymbol{v}_{1} \cdot \boldsymbol{v}_{2}\right)$ that guarantees the Lorentz invariance of the product of the number densities can also be written as

$1-\boldsymbol{v}_{1} \cdot \boldsymbol{v}_{2}=\frac{\gamma_{\mathrm{r}}}{\gamma_{1} \gamma_{2}}=\frac{p_{1} \cdot p_{2}}{E_{1} E_{2}}$

where $\gamma_{\mathrm{r}}=1 / \sqrt{1-v_{\mathrm{rel}}^{2}}$ is the Lorentz factor associated with $v_{\text {rel }}$ and $\gamma_{i}$ the Lorentz factors associated with $\boldsymbol{v}_{i}$.

If the element of Lorentz invariant phase space is defined as usual

$\mathrm{d} \tilde{p}_{i}=\frac{\mathrm{d}^{3} \boldsymbol{p}_{i}}{(2 \pi)^{3} 2 E_{i}}$

and one particle states for bosons and fermions are normalized to $2 E_{i}$ such that the density per unit volume is $2 E_{i}$, then, using (B.10), the flux (B.9) simplifies to

$F=4\left(p_{1} \cdot p_{2}\right) v_{\text {rel }}$

Substituting the expression of $v_{\text {rel }}$ in the momentum representation, formula (B.7), in Eq. (B.12), the scalar product $p_{1} \cdot p_{2}$ cancels out and the standard explicit form is recovered

$F=4 \sqrt{\left(p_{1} \cdot p_{2}\right)^{2}-m_{1}^{2} m_{2}^{2}}$ 
Appendix B.3: Cross section and collision integral

The integrated collision term of the Boltzmann equation, neglecting quantum effects, can be written as

$$
\int \prod_{i=1}^{4} \mathrm{~d} \tilde{p}_{i}\left[f_{3} f_{4} W(3,4 \mid 1,2)-f_{1} f_{2} W(1,2 \mid 3,4)\right],
$$

where $W(i j \mid k l)=(2 \pi)^{4} \delta^{4}\left(P_{i j}-P_{k l}\right) \sum_{s_{i}, s_{f}}\left|\mathcal{M}_{i j \rightarrow k l}\right|^{2}$, and $f_{i}$ is the phase-space distribution.

Using the unitary condition $\int \mathrm{d} \tilde{p}_{3} \mathrm{~d} \tilde{p}_{4} W(3,4 \mid 1,2)=$ $\int \mathrm{d} \tilde{p}_{3} \mathrm{~d} \tilde{p}_{4} W(1,2 \mid 3,4)$ to write the collision integral only in terms of the annihilation rate

$$
\frac{1}{1+\delta_{12}} \int \prod_{i=1}^{4} \mathrm{~d} \tilde{p}_{i}\left(f_{3} f_{4}-f_{1} f_{2}\right) W(1,2 \mid 3,4)
$$

we keep out a statistical factor accounting for the possibility of identical particles.

By definition, the invariant cross section, using the flux in the form (B.12), is

$\sigma=\frac{1}{4\left(p_{1} \cdot p_{2}\right) v_{\text {rel }}} \int \mathrm{d} \tilde{p}_{3} d \tilde{p}_{4} \frac{W(1,2 \mid 3,4)}{g_{1} g_{2}}$,

$g_{i}=\left(2 s_{i}+1\right)$ being for the spin degrees of freedom.

Assuming as usual that the annihilation products are described by the equilibrium phase-space distribution at zero chemical potential $f_{0, i}$, we have $f_{3} f_{4}=f_{0,3} f_{0,4}=f_{0,1} f_{0,2}$, the last equality following from energy conservation. Hence

$\frac{g_{1} g_{2}}{1+\delta_{12}} \int \prod_{i=1}^{2} \frac{\mathrm{d}^{3} \boldsymbol{p}_{i}}{(2 \pi)^{3} E_{i}}\left(p_{1} \cdot p_{2}\right)\left(f_{0,1} f_{0,2}-f_{1} f_{2}\right) \sigma v_{\text {rel }}$.

The equilibrium phase-space distribution $f_{0, i}$ is related to the number density $n_{0}$ and to the momentum distribution $f_{0, p}(\boldsymbol{p})$ by $g_{i} /(2 \pi)^{3} f_{0, i}=n_{0, i} f_{0, p}(\boldsymbol{p})$. Assuming further that the non-equilibrium phase-space function at finite chemical potential $f_{i}$ remains proportional to the equilibrium momentum distribution by a factor given by the nonequilibrium number density $n_{i}, g_{i} /(2 \pi)^{3} f_{i}=n_{i} f_{0, p}(\boldsymbol{p})$, we obtain

$$
\frac{1}{1+\delta_{12}}\left(n_{0,1} n_{0,2}-n_{1} n_{2}\right)\left\langle\sigma v_{\text {rel }}\right\rangle
$$

When the species 1 and 2 are the same, it takes the usual form $\left\langle\sigma v_{\text {rel }}\right\rangle\left(n_{0}^{2}-n^{2}\right)$ with the factor $1 / 2$ canceled by stoichiometric coefficient appearing in the left-hand side of the complete kinetic equation; see for example Ref. [32].
Appendix B.4: Averaged thermal rate

In Eq. (B.16) the general definition of relativistic thermal averaged rate is

$$
\left\langle\sigma v_{\mathrm{rel}}\right\rangle=\int \prod_{i=1}^{2} \frac{\mathrm{d}^{3} \boldsymbol{p}_{i}}{E_{i}}\left(p_{1} \cdot p_{2}\right) f_{0, p}\left(\boldsymbol{p}_{1}\right) f_{0, p}\left(\boldsymbol{p}_{2}\right) \sigma v_{\text {rel }} .
$$

In the case of the relativistic Maxwell-Boltzmann-Juttner statistics, the momentum distribution is

$$
f_{0, p}(\boldsymbol{p})=\frac{1}{4 \pi m^{2} T K_{2}(x)} e^{-\sqrt{\boldsymbol{p}^{2}+m^{2}} / T},
$$

and as shown in Ref. [27], the six-dimensional integral on the right-hand side of Eq. (B.17) reduces to

$\left\langle\sigma v_{\text {rel }}\right\rangle=\int_{0}^{1} \mathrm{~d} v_{\text {rel }} \mathcal{P}\left(v_{\text {rel }}\right) \sigma v_{\text {rel }}$,

where the probability distribution of $v_{\text {rel }}$, for example for $m_{1}=m_{2}=m$, is

$\mathcal{P}\left(v_{\mathrm{rel}}\right)=\frac{x}{\sqrt{2} K_{2}^{2}(x)} \frac{\gamma_{\mathrm{r}}^{3}\left(\gamma_{\mathrm{r}}^{2}-1\right)}{\sqrt{\gamma_{\mathrm{r}}+1}} K_{1}\left(\sqrt{2} x \sqrt{\gamma_{\mathrm{r}}+1}\right)$.

This is completely analogous to the nonrelativistic case where the probability distribution of $v_{r}$, for $m_{1}=m_{2}=m$, is

$P\left(v_{r}\right)=\sqrt{\frac{2}{\pi}} x^{3 / 2} v_{r}^{2} e^{-x \frac{v_{r}^{2}}{4}}$,

and the thermal average reads

$\left\langle\sigma_{\mathrm{nr}} v_{r}\right\rangle_{\mathrm{nr}}=\int_{0}^{\infty} \mathrm{d} v_{r} P\left(v_{r}\right) \sigma_{\mathrm{nr}} v_{r}$.

Given the total annihilation cross section $\sigma$ the product $\sigma v_{\text {rel }}$ will reduce to the nonrelativistic limit $\sigma_{\mathrm{nr}} v_{r}$ and $\left\langle\sigma v_{\text {rel }}\right\rangle$ to $\left\langle\sigma_{\mathrm{nr}} v_{r}\right\rangle_{\mathrm{nr}}$ in the COF when $v_{\mathrm{rel}} \sim v_{r} \ll 1$. Expressing Eq. (B.19) in terms of $s$ using (B.8), we obtain the usual integral $[27,36]$ useful for practical calculation

$$
\begin{aligned}
\left\langle\sigma v_{\text {rel }}\right\rangle= & \frac{1}{8 T \prod_{i} m_{i}^{2} K_{2}\left(x_{i}\right)} \\
& \times \int_{M^{2}}^{\infty} \mathrm{d} s \frac{\lambda\left(s, m_{1}^{2}, m_{2}^{2}\right)}{\sqrt{s}} K_{1}\left(\frac{\sqrt{s}}{T}\right) \sigma,
\end{aligned}
$$

with $x_{i}=m_{i} / T$ and $M=\left(m_{1}+m_{2}\right)$.

We have recently become aware of the paper [37] where, probably for the first time, the thermal average of relativistic 
rates was discussed and it was realized that with the relativistic Maxwell-Boltzmann statistics formula (B.17) reduces to a single integral over the distribution over the relative momentum. With some algebra and change of variables it is easy to verify that for example Eqs. (11b) and (12a) of [37] coincide with Eqs. (29) and (37) of Ref. [27]. In Ref. [37] the cases of collisions of two massive particles, two massless particles and a massive with a massless particles are treated separately as if different definitions of flux and cross sections were necessary in each case. Clearly this distinction is unnecessary for the formulation we have given is completely general and valid in any case. We finally note that an integral formula similar to (B.23) was also given in Ref. [29].

\section{Appendix B.5: No need for the Møller velocity}

By noting that in Eq. (B.9) the factor $\left(1-\boldsymbol{v}_{1} \cdot \boldsymbol{v}_{2}\right)$ can cancel the same factor in the denominator of $v_{\text {rel }}$, the invariant flux can also be written in the form

$F=n_{1} n_{2} \sqrt{\left(\boldsymbol{v}_{1}-\boldsymbol{v}_{2}\right)^{2}-\left(\boldsymbol{v}_{1} \times \boldsymbol{v}_{2}\right)^{2}}$.

In the textbook by Landau and Lifschits [38] this form is attributed to Pauli without giving any reference, while its origin is more generally attributed to Møller [39].

It is interesting to look at original paper by Møller [39]. With our notation, he wants to prove that the flux given (B.24) is invariant. In order to do that he shows that this can be written as a product of two invariant quantities: the ratio $\frac{n_{1} n_{2}}{E_{1} E_{2}}$ and the quantity $B=\sqrt{\left(p_{1} \cdot p_{2}\right)^{2}-m_{1}^{2} m_{2}^{2}}$ and there he stops.

The flux factor written in the form (B.24) has the same structure of thee nonrelativistic expression $n_{1} n_{2} v_{r}$. Probably for this reason it has been later introduced in the literature the notion of Møller velocity

$\bar{v}=\sqrt{\left(\boldsymbol{v}_{1}-\boldsymbol{v}_{2}\right)^{2}-\left(\boldsymbol{v}_{1} \times \boldsymbol{v}_{2}\right)^{2}}=\frac{\sqrt{\left(p_{1} \cdot p_{2}\right)^{2}-m_{1}^{2} m_{2}^{2}}}{E_{1} E_{2}}$.

It is worth to stress that neither Møller nor Landau and Lifschits attribute any particular meaning to Eq. (B.25) and do not define it as a particular velocity, even less as relative velocity. Clearly $\bar{v}$ is nothing but the numerator of the formula defining $v_{\text {rel }}$ because $\bar{v}=\left(1-\boldsymbol{v}_{1} \cdot \boldsymbol{v}_{2}\right) v_{\text {rel }}$, where the factor $\left(1-\boldsymbol{v}_{1} \cdot \boldsymbol{v}_{2}\right)$ comes from the definition of the invariant flux (B.9). Already this fact indicates that $\bar{v}$ is not a fundamental physical quantity and overall, it is not the relative velocity, nor when the velocities are collinear.

On the contrary, in DM literature and in textbooks, when defining the flux factor for the relativistic invariant cross section, it is incorrectly asserted that in a frame where the veloci- ties are collinear the quantity $\left|\boldsymbol{v}_{1}-\boldsymbol{v}_{2}\right|$ is the relative velocity, while in a generic frame is given by (B.25). The form (B.24) of the flux is a simple consequence of the fundamental quantities (B.5) and (B.9), there is no new physics or concept in it. For these reasons, and for its non-invariant and non-physical nature, $\bar{v}$ should not be used.

\section{References}

1. A. Kurylov, M. Kamionkowski, Generalized analysis of weakly interacting massive particle searches. Phys. Rev. D 69, 063503 (2004). arXiv:hep-ph/0307185

2. J. Fan, M. Reece, L.T. Wang, Non-relativistic effective theory of dark matter direct detection. JCAP 1011, 042 (2010). arXiv: 1008.1591

3. A.L. Fitzpatrick, W. Haxton, E. Katz, N. Lubbers, Y. Xu, The effective field theory of dark matter direct detection. JCAP 1302, 004 (2013). arXiv:1203.3542

4. M. Beltran, D. Hooper, E.W. Kolb, Z.C. Krusberg, Deducing the nature of dark matter from direct and indirect detection experiments in the absence of collider signatures of new physics. Phys. Rev. D 80, 043509 (2009). arXiv:0808.3384

5. H. Zhang, Q.H. Cao, C.R. Chen, C.S. Li, Effective dark matter model: relic density, CDMS II, Fermi LAT and LHC. JHEP 1108, 018 (2011). arXiv:0912.4511

6. M.R. Buckley, Asymmetric dark matter and effective operators. Phys. Rev. D 84, 043510 (2011). arXiv:1104.1429

7. S. Matsumoto, S. Mukhopadhyay, Y.L.S. Tsai, Singlet Majorana fermion dark matter: a comprehensive analysis in effective field theory. JHEP 1410, 155 (2014). arXiv: 1407.1859

8. M.A. Fedderke, J.Y. Chen, E.W. Kolb, L.T. Wang, The fermionic dark matter higgs portal: an effective field theory approach. JHEP 1408, 122 (2014). arXiv: 1404.2283

9. J.Y. Chen, E.W. Kolb, L.T. Wang, Dark matter coupling to electroweak gauge and Higgs bosons: an effective field theory approach. Phys. Dark Univ. 2, 200 (2013). arXiv:1305.0021

10. A. Berlin, D. Hooper, S.D. McDermott, Simplified Dark matter models for the galactic center gamma-ray excess. Phys. Rev. D 89, 115022 (2014). arXiv: 1404.0022

11. S. Chang, R. Edezhath, J. Hutchinson, M. Luty, Effective WIMPs. Phys. Rev. D 89, 015011 (2014). arXiv: 1307.8120

12. C. Balzs, T. Li, J.L. Newstead, Thermal dark matter implies new physics not far above the weak scale. JHEP 1408, 061 (2014). arXiv: 1403.5829

13. K. Cheung, P.Y. Tseng, Y.L.S. Tsai, T.C. Yuan, Global constraints on effective dark matter interactions: relic density, direct detection, indirect detection, and collider. JCAP 1205, 001 (2012). arXiv: 1201.3402

14. J. Goodman, M. Ibe, A. Rajaraman, W. Shepherd, T.M.P. Tait, H.B. $\mathrm{Yu}$, Constraints on light majorana dark matter from colliders. Phys. Lett. B 695, 185 (2011). arXiv:1005.1286

15. J. Goodman, M. Ibe, A. Rajaraman, W. Shepherd, T.M.P. Tait, H.B. Yu, Constraints on dark matter from colliders. Phys. Rev. D 82, 116010 (2010). arXiv: 1008.1783

16. O. Buchmueller, M.J. Dolan, C. McCabe, Beyond effective field theory for dark matter searches at the LHC. JHEP 1401, 025 (2014). arXiv: 1308.6799

17. G. Busoni, A. De Simone, E. Morgante, A. Riotto, On the validity of the effective field theory for dark matter searches at the LHC. Phys. Lett. B 728, 412 (2014). arXiv:1307.2253

18. G. Busoni, A. De Simone, J. Gramling, E. Morgante, A. Riotto, On the validity of the effective field theory for dark matter searches at 
the LHC, part II: complete analysis for the s-channel. JCAP 1406, 060 (2014). arXiv: 1402.1275

19. G. Busoni, A. De Simone, T. Jacques, E. Morgante, A. Riotto, On the validity of the effective field theory for dark matter searches at the LHC part III: analysis for the t-channel. JCAP 1409, 022 (2014). arXiv:1405.3101

20. J.M. Zheng, Z.H. Yu, J.W. Shao, X.J. Bi, Z. Li, H.H. Zhang, Constraining the interaction strength between dark matter and visible matter: I. Fermionic dark matter. Nucl. Phys. B 854, 350 (2012). arXiv: 1012.2022

21. H. Dreiner, M. Huck, M. Krmer, D. Schmeier, J. Tattersall, Illuminating dark matter at the ILC. Phys. Rev. D 87, 075015 (2013). arXiv: 1211.2254

22. J. Blumenthal, P. Gretskov, M. Krmer, C. Wiebusch, Effective field theory interpretation of searches for dark matter annihilation in the Sun with the IceCube Neutrino Observatory. Phys. Rev. D 91, 035002 (2015). arXiv:1411.5917

23. G. Busoni, A. De Simone, T. Jacques, E. Morgante, A. Riotto, Making the most of the relic density for dark matter searches at the LHC 14 TeV run. JCAP 1503, 022 (2015). arXiv:1410.7409

24. C.L. Bennett et al., [WMAP Collaboration], Nine-year wilkinson microwave anisotropy probe (WMAP) observations: final maps and results. Astrophys. J. Suppl. 208, 20 (2013). arXiv:1212.5225

25. P.A.R. Ade et al., [Planck Collaboration], Planck 2013 results. XVI. Cosmological parameters. Astron. Astrophys. 571, A16 (2014). arXiv: 1303.5076

26. P. Gondolo, G. Gelmini, Cosmic abundances of stable particles: improved analysis. Nucl. Phys. B 360, 145 (1991)

27. M. Cannoni, Relativistic $\left\langle\sigma v_{\text {rel }}\right\rangle$ in the calculation of relics abundances: a closer look. Phys. Rev. D 89, 103533 (2014). arXiv:1311.4494, arXiv:1311.4508
28. M. Drees, M. Kakizaki, S. Kulkarni, The thermal abundance of semi-relativistic relics. Phys. Rev. D 80, 043505 (2009). arXiv:0904.3046

29. M. Claudson, L.J. Hall, I. Hinchliffe, Cosmological baryon generation at low temperatures. Nucl. Phys. B 241, 309 (1984)

30. M. Srednicki, R. Watkins, K.A. Olive, Calculations of relic densities in the early universe. Nucl. Phys. B 310, 693 (1988)

31. K. Griest, D. Seckel, Three exceptions in the calculation of relic abundances. Phys. Rev. D 43, 3191 (1991)

32. M. Cannoni, Exact theory of freeze out. Eur. Phys. J. C 75, 106 (2015). arXiv:1407.4108

33. G. Steigman, B. Dasgupta, J.F. Beacom, Precise relic WIMP abundance and its impact on searches for dark matter annihilation. Phys. Rev. D 86, 023506 (2012). arXiv: 1204.3622

34. M. Drees, F. Hajkarim, E.R. Schmitz, The effects of QCD equation of state on the relic density of WIMP dark matter. JCAP 1506, 025 (2015). arXiv: 1503.03513

35. R.J. Scherrer, M.S. Turner, On the relic, cosmic abundance of stable weakly interacting massive particles. Phys. Rev. D 33, 1585 (1986)

36. J. Edsjo, P. Gondolo, Neutralino relic density including coannihilations. Phys. Rev. D 56, 1879 (1997). arXiv:hep-ph/9704361

37. T.A. Weaver, Reaction rates in a relativistic plasma. Phys. Rev. A 13, 1563 (1976)

38. L.D. Landau, E.M. Lifschits, The Classical Theory of Fields: Course of Theoretical Physics, vol. 2 (Pergamon Press, New York, 1975)

39. C. Møller, General Properties of the Characteristic Matrix in the Theory of Elementary Particles. Det kgl. Danske Videnskabernes Selskab. Matematisk-fysiske Meddelelser, vol. 23, No. 1 (1945) 